\title{
GENERALIZED SECOND-ORDER DERIVATIVES OF CONVEX FUNCTIONS IN REFLEXIVE BANACH SPACES
}

\author{
CHI NGOC DO
}

\begin{abstract}
Generalized second-order derivatives introduced by Rockafellar in finite-dimensional spaces are extended to convex functions in reflexive Banach spaces. Parallel results are shown in the infinite-dimensional case. A result that plays an important role in applications is that the generalized second-order differentiability is preserved under the integral sign.
\end{abstract}

Generalized second-order derivatives in nonsmooth analysis have drawn much attention in the past few years because of their importance in sensitivity analysis and in statements of optimality conditions. Many authors have tried to define second-order directional derivatives in quite different ways. Most definitions have been confined to finite-valued functions; see for example. [3], [4], [20] for nonconvex and [9] for convex functions. Recently, R. T. Rockafellar in [13] has introduced second-order derivatives for extended real-valued functions in $\mathbf{R}^{n}$ based on the epi-convergence of second-order difference quotients and has called them epi-derivatives. He showed that for a very broad class of extended-real-valued functions which includes most of the functions in nonlinear programming, such second-order epi-derivatives exist and are given by usable formulas. Second-order optimality conditions in nonlinear programming have been obtained in terms of epi-derivatives; see [17]. In the convex case, the theory of second-order epi-derivatives has special potential. It helps in creating a foundation for sensitivity analysis in convex optimization and was worked out along such lines in [14].

In this paper, we shall extend Rockafellar's ideas to the case of infinite dimensional spaces in the convex analysis framework. Such generalization has been attempted by Ndoutoume in [11] with considerably more restrictive assumptions. The class of functions studied in his paper turns out to include only "generalized purely quadratic functions."

Before going to our theory of second-order derivatives, we need to review some notion of epi-convergence. For a thorough treatment of this convergence and other applications, we refer the readers to [1,2].

Throughout the paper, $E$ and $F$ are reflexive Banach spaces. The norm topology (strong topology) and weak topology are abbreviated by letters $s$ and $w$ respectively.

Received by the editors October 30, 1990.

1980 Mathematics Subject Classification (1985 Revision). Primary 49A50; Secondary 49A51, $58 \mathrm{C} 20$.

Key words and phrases. Generalized second-order derivatives, epi-convergence, Mosco convergence, epi-derivatives, proto-derivatives, integral functionals, normal integrands. 


\section{EPI-CONVERGENCE}

Definition 1.1. Let $\left(S_{n}\right)$ be a sequence of subsets in $E$. The Kuratowski limits in the strong topology are defined by

(1.1) $s$-lim inf $S_{n}=\left\{x \in E: \exists x_{n} \in S_{n}\right.$ for $n$ sufficiently large, with $\left.x_{n} \stackrel{s}{\rightarrow} x\right\}$,

$$
\text { s-lim } \sup S_{n}=\left\{x \in E: \exists\left(n_{k}\right)_{k \in \mathbf{N}}, \exists x_{k} \in S_{n_{k}} \text { with } x_{k} \stackrel{s}{\rightarrow} x\right\} .
$$

Obviously, s-lim $\inf S_{n} \subset \operatorname{s-lim} \sup S_{n}$. The sequence $S_{n}$ is said to be convergent (in strong topology) if s-lim inf $S_{n}=\mathrm{s}-\lim \sup S_{n}$, and the common value $S$ is written as $S=\mathrm{s}-\lim S_{n}$. It is necessary that $S$ be closed.

Salinetti and Wets in $[18,19]$ gave some characterizations for a sequence of closed sets $S_{n}$ to converge to a closed set $S$ in a finite-dimensional space. Among those one has

$$
S_{n} \rightarrow S \Leftrightarrow d\left(x, S_{n}\right) \rightarrow d(x, S) \quad \forall x \in E,
$$

where $d(x, S)$ is the distance from $x$ to the set $S$. In any Banach space, the condition $d\left(x, S_{n}\right) \rightarrow d(x, S)$ for all $x \in E$ always implies that $S_{n} \stackrel{s}{\rightarrow} S$.

The limits in the weak topology corresponding to (1.1) and (1.2) are

w-lim inf $S_{n}=\left\{x \in E: \exists x_{n} \in S_{n}\right.$ for $n$ sufficiently large, with $\left.x_{n} \stackrel{w}{\rightarrow} x\right\}$,

$$
\text { w-lim } \sup S_{n}=\left\{x \in E: \exists\left(n_{k}\right)_{k \in \mathbf{N}}, \exists x_{k} \in S_{n_{k}} \text { with } x_{k} \stackrel{w}{\rightarrow} x\right\} .
$$

Actually, these should be termed the "sequential w-lim inf" and "sequential w-lim sup." It is also obvious that

$$
\text { s-lim inf } S_{n} \subset \text { w-lim } \inf S_{n}, \quad \text { s-lim } \sup S_{n} \subset \mathrm{w}-\lim \sup S_{n} .
$$

The sequence $\left(S_{n}\right)$ is said to be Mosco convergent if w-lim $\sup S_{n} \subset \mathrm{s}-\lim \inf S_{n}$, or in other words, all four sets above are equal:

$$
\mathrm{s}-\lim \inf S_{n}=\mathrm{w}-\lim \inf S_{n}=\mathrm{s}-\lim \sup S_{n}=\mathrm{w}-\lim \sup S_{n} .
$$

The common value $S$ is then denoted by $\operatorname{M}-\lim S_{n}$.

Definition 1.2. Let $\varphi_{n}, \varphi: E \rightarrow \overline{\mathbf{R}},(n \in \mathbf{N})$. The sequence $\varphi_{n}$ is said to Mosco epi-converge (or simply Mosco converge) to $\varphi$, written $\varphi=\mathrm{M}-e-\lim \varphi_{n}$ (or $\mathrm{M}-\lim \varphi_{n}$ ), if the epigraphs epi $\varphi_{n}$ (in $E \times \mathbf{R}$ ) Mosco converge to epi $\varphi$. If $E$ is finite-dimensional, then the weak and strong topologies coincide and the Mosco convergence is simply the epi-convergence.

For an arbitrary sequence $\varphi_{n}$, the function whose epigraph is $s-\lim \inf \left(\operatorname{epi} \varphi_{n}\right)$ is denoted (customarily) by $e_{s}$-ls $\varphi_{n}$. One has

$$
\left(e_{s}-\operatorname{ls} \varphi_{n}\right)(\xi)=\inf _{\xi_{n} s \xi}\left\{\lim \sup \varphi_{n}\left(\xi_{n}\right)\right\} .
$$

The infimum is actually attained (cf. [1, Theorem 1.13]). Similarly, the function whose epigraph is $w$-lim $\sup \left(\operatorname{epi} \varphi_{n}\right)$ is given by

$$
\left(e_{w}-\operatorname{li} \varphi_{n}\right)(\xi)=\inf _{\xi_{n} \rightarrow \xi}\left\{\liminf \varphi_{n}\left(\xi_{n}\right)\right\} .
$$

The infimum is not attained in general (since the weak topology need not be 
first countable). Mosco (epi-) convergence of $\varphi_{n}$ to $\varphi$ is then characterized by $e_{s}-\operatorname{ls} \varphi_{n} \leq \varphi \leq e_{w}-$ li $\varphi_{n}$, or in other words

$$
\begin{array}{ll}
\forall \xi_{n} \stackrel{w}{\rightarrow} \xi, & \varphi(\xi) \leq \lim \inf \varphi_{n}\left(\xi_{n}\right), \\
\exists \xi_{n} \stackrel{s}{\rightarrow} \xi, & \varphi(\xi) \geq \lim \sup \varphi_{n}\left(\xi_{n}\right) .
\end{array}
$$

Equality then holds in (1.8).

What we will be dealing with is a family of functions $\left(\varphi_{t}\right)$ parameterized by $t>0$. The Mosco epi-convergence of $\varphi_{t}$ to $\varphi$ as $t \downarrow 0$ is defined in a natural way by saying $\varphi_{t_{n}} \stackrel{M}{\rightarrow} \varphi$ for every sequence $t_{n} \downarrow 0$. In view of (1.7) and (1.8), this says that: for every sequence $t_{n} \downarrow 0$, for every $\xi$,

$$
\begin{array}{ll}
\forall \xi_{n} \stackrel{w}{\rightarrow} \xi, & \varphi(\xi) \leq \liminf \varphi_{t_{n}}\left(\xi_{n}\right), \\
\exists \xi_{n} \stackrel{s}{\rightarrow} \xi, & \varphi(\xi) \geq \lim \sup \varphi_{t_{n}}\left(\xi_{n}\right) .
\end{array}
$$

Equality then holds in $(1.10)$ and $\varphi(\xi)=\lim \varphi_{t_{n}}\left(\xi_{n}\right)$.

One may define directly the limits of a family of sets $\left(S_{t}\right)_{t>0}$ by

s-lim inf $S_{t}=\left\{x: \forall t_{n} \downarrow 0, \exists x_{n} \stackrel{s}{\rightarrow} x ;\right.$ where $x_{n} \in S_{t_{n}}$ for $n$ sufficiently large $\}$,

$\mathrm{w}$-lim inf $S_{t}=\left\{x: \forall t_{n} \downarrow 0, \exists x_{n} \stackrel{w}{\rightarrow} x\right.$ where $x_{n} \in S_{t_{n}}$ for $n$ sufficiently large $\}$,

$$
\mathrm{w}-\lim \sup S_{t}=\left\{x: \exists t_{n} \downarrow 0, \exists x_{n} \stackrel{w}{\rightarrow} x \text { with } x_{n} \in S_{t_{n}}\right\} \text {. }
$$

One says that $S_{t}$ (strongly) converges to $S$ as $t \downarrow 0$ if

$$
\text { s-lim } \sup S_{t} \subset S \subset \mathrm{s}-\lim \inf S_{t}
$$

(then all three sets are equal), and $S_{t}$ Mosco converges to $S$ as $t \downarrow 0$ if

$$
\text { w-lim } \sup S_{t} \subset S \subset \mathrm{s}-\lim \inf S_{t},
$$

then all four sets in (1.11)-(1.14) are equal. It can be seen that

$$
S_{t} \rightarrow S(t \downarrow 0) \Leftrightarrow S_{t_{n}} \rightarrow S(n \rightarrow \infty), \quad \forall t_{n} \downarrow 0 .
$$

Therefore the Mosco epi-convergence of a family of functions $\left(\varphi_{t}\right)_{t>0}$ to $\varphi$ as defined via sequences is the same as the Mosco convergence of the family $\left(\right.$ epi $\left.\varphi_{t}\right)$ to epi $\varphi$.

The following proposition is immediate:

Proposition 1.3. Let $\varphi_{t}$ Mosco epi-converge to $\varphi$. If the functions $\left(\varphi_{t}\right)_{t>0}$ are lower semicontinuous convex, then so is $\varphi$.

Remark 1.4. In the proposition above, convexity implies that the lower semicontinuity is in both the weak and strong topologies.

If all $\varphi_{t}$ are proper, it is not necessary that $\varphi$ be proper. This is seen by the following example. Let

$$
\varphi_{t}(\xi)=\left\{\begin{array}{ll}
-\xi / t^{2} & \text { if } \xi \in[0, t], \\
\infty & \text { otherwise, }
\end{array} \quad \varphi(\xi)= \begin{cases}-\infty & \text { if } \xi=0 \\
\infty & \text { otherwise }\end{cases}\right.
$$


Then $\varphi_{t}$ is a closed proper convex function and $\varphi_{t}(0)=0$ for every $t>0$, and $\varphi_{t} \stackrel{M}{\rightarrow} \varphi$, but $\varphi$ is not proper.

\section{SECOND-ORDER EPI-DERIVATIVES}

Throughout this section, $f$ denotes a closed proper convex function, $f$ : $E \rightarrow \overline{\mathbf{R}}$.

Definition 2.1. Let $f$ be finite at $x \in E$. Let $z \in E^{*}$ and consider the secondorder difference quotient functions

$$
\left(\Delta_{t}^{(2)} f\right)_{x, z}(\xi):=\frac{2}{t^{2}}\{f(x+t \xi)-f(x)-t\langle z, \xi\rangle\}, \quad \xi \in E(t>0) .
$$

If these functions Mosco epi-converge (as $t \downarrow 0$ ) to some function $\varphi$ having $\varphi(0) \neq-\infty$, then we say that $f$ is twice epi-differentiable at $x$ relative to $z$, and $\varphi$ is called the second-order epi-derivative of $f$ at $x$ relative to $z$. We then write $f_{x, z}^{\prime \prime}$ instead of $\varphi$.

One can see easily that the above differentiability is a local property, i.e. it depends only on the values of $f$ in a neighborhood of $x$.

The constant 2 on the right-hand side of (2.1) is introduced to make our definition of generalized second-order derivative "closer" to those in classical sense. In fact, a Taylor expansion for a function $f$ of class $\mathscr{C}^{2}$ around $x$ shows that the difference quotient with $z=D f(x)$ converges pointwise to $\left\langle D^{2} f(x) \xi, \xi\right\rangle$. In trade off for this classical meaning, the constant $\frac{1}{2}$ will pop up in our later formulas.

Proposition 2.2. The second-order epi-derivative $f_{x, z}^{\prime \prime}$ is closed, proper convex, positively homogeneous of degree 2 and $f_{x, z}^{\prime \prime}(0)=0$.

Proof. The verification is straightforward as in the finite-dimensional case; see [17].

Proposition 2.3. If $f$ is twice epi-differentiable at $x$ relative to $z$, then $z \in$ $\partial f(x)$, the subdifferential of $f$ at $x$. Furthermore, one has $f_{x, z}^{\prime \prime} \geq 0$ and $0 \in \partial f_{x, z}^{\prime \prime}(0)$.

Proof. For a convex function $f$ which is finite at $x$, one has

$$
z \in \partial f(x) \Leftrightarrow \exists \varepsilon>0, \forall x^{\prime} \in B(x, \varepsilon), \quad f\left(x^{\prime}\right) \geq f(x)+\left\langle z, x^{\prime}-x\right\rangle .
$$

Now suppose that $f$ is twice epi-differentiable at $x$ relative to $z$ and that $z \notin \partial f(x)$. Then by (2.2), there exists a sequence $\xi_{n} \stackrel{s}{\rightarrow} 0$ such that

$$
\varphi\left(\xi_{n}\right):=f\left(x+\xi_{n}\right)-f(x)-\left\langle z, \xi_{n}\right\rangle=\alpha_{n}<0 .
$$

The function $\varphi$ defined in this way (for every $\xi$ ) is lower semicontinuous, convex and $\varphi(0)=0$, hence

$$
0=\varphi(0) \leq \liminf \varphi\left(\xi_{n}\right)=\liminf \alpha_{n} \leq \lim \sup \alpha_{n} \leq 0 .
$$

Thus $\lim \alpha_{n}=0$. We may assume that $\left|\alpha_{n}\right|<1$ for all $n$.

The convexity of $\varphi$ implies that

$$
\varphi\left(-\alpha_{n} \xi_{n}\right) \leq\left(1+\alpha_{n}\right) \varphi(0)-\alpha_{n} \varphi\left(\xi_{n}\right)=-\alpha_{n} \varphi\left(\xi_{n}\right)=-\alpha_{n}^{2} .
$$


The Mosco convergence of $\left(\Delta_{t}^{(2)} f\right)_{x, z}$ to $f_{x, z}^{\prime \prime}$ gives (by (1.9) with $t_{n}=-\alpha_{n}>$ $0)$,

$$
\begin{aligned}
0 & =f_{x, z}^{\prime \prime}(0) \leq 2 \liminf \frac{f\left(x-\alpha_{n} \xi_{n}\right)-f(x)+\alpha_{n}\left\langle z, \xi_{n}\right\rangle}{\left(-\alpha_{n}\right)^{2}} \\
& =2 \liminf \frac{\varphi\left(-\alpha_{n} \xi_{n}\right)}{\left(\alpha_{n}\right)^{2}} \leq 2 \liminf \frac{-\alpha_{n}^{2}}{\alpha_{n}^{2}}=-2
\end{aligned}
$$

a contradiction. Therefore we must have $z \in \partial f(x)$. This also implies that $\varphi \geq 0$ and hence $f_{x, z}^{\prime \prime} \geq 0$. By Proposition 2.2, 0 is a minimum point of $f_{x, z}^{\prime \prime}$, thus $0 \in \partial f_{x, z}^{\prime \prime}(0)$.

Definition 2.4. (a) $f$ is said to be twice epi-differentiable at $x$ if $\partial f(x) \neq \varnothing$ and $f$ is twice epi-differentiable at $x$ relative to $z$ for every $z \in \partial f(x)$.

(b) $f$ is essentially twice epi-differentiable (not referring to any particular $x$ ) if for every $x$ at which $\partial f(x) \neq \varnothing, f$ is twice epi-differentiable at $x$.

(c) $f$ is twice epi-differentiable if for every $x$ at which $f(x)$ is finite, $f$ is twice epi-differentiable at $x$.

One can prove directly that if a convex function $f$ is $\mathscr{C}^{2}$ (Fréchet) in a neighborhood of $x$, then it is twice epi-differentiable at $x$; see [6]. However, we will provide an indirect proof later, based on a result in the next section, where the continuity of the second-order derivative mapping is not needed.

Theorem 2.5 (Conjugacy). For a closed proper convex function $f$, one has

(a) $z \in \partial f(x) \Leftrightarrow x \in \partial f^{*}(z)$.

(b) $f$ is twice epi-differentiable at $x$ relative to $z$ if and only if $f^{*}$ is twice epi-differentiable at $z$ relative to $x$. One has $\frac{1}{2}\left(f_{z}^{*}, x\right)^{\prime \prime}=\left(\frac{1}{2} f_{x, z}^{\prime \prime}\right)^{*}$.

(Recall that the conjugate $f^{*}$ of $f$ is defined by

$$
f^{*}(z)=\sup _{x \in E}\{\langle x, z\rangle-f(x)\}, z \in E^{*} \text {.) }
$$

Proof. Part (a) is just a well-known fact in convex analysis. For part (b), one can verify easily that $\left(\frac{1}{2} \Delta_{t}^{(2)} f\right)_{x, z}^{*}=\frac{1}{2}\left(\Delta_{t}^{(2)} f^{*}\right)_{z, x}$, where $\Delta_{t}^{(2)} f$ and $\Delta_{t}^{(2)} f^{*}$ are the second-order difference quotients of $f$ and $f^{*}$ respectively. Therefore, part (b) is a consequence of Wijsman-Mosco's theorem on the continuity of the Legendre-Fenchel transform (cf. [1, Theorem 3.18]), which says that for a sequence of closed proper convex functions $\left(\varphi_{n}\right), \varphi$, one has $\varphi_{n} \stackrel{M}{\rightarrow} \varphi \Leftrightarrow \varphi_{n}^{*} \stackrel{M}{\rightarrow}$ $\varphi^{*}$.

Corollary 2.6. $f$ is essentially twice epi-differentiable if and only if $f^{*}$ is.

Let $C$ be a closed convex set in $E$ and $u \in C$. Recall that the normal cone $N_{C}(u)$ is defined by

$$
N_{C}(u)=\left\{\xi \in E^{*}:\langle\xi, v-u\rangle \leq 0, \forall v \in C\right\}
$$

and the tangent cone $T_{C}(u)$ is the polar of the normal cone

$$
T_{C}(u)=N_{C}(u)^{\circ}=\left\{\eta \in E:\langle\eta, \xi\rangle \leq 0, \forall \xi \in N_{C}(u)\right\} .
$$

The tangent cone is also called the support cone elsewhere. One has

$$
T_{C}(u)=\operatorname{cl}\{\eta: \exists \lambda>0 \text { such that } u+\lambda \eta \in C\} .
$$


In terms of limits of sets, one has

$$
T_{C}(u)=\limsup _{t \downarrow 0} \frac{1}{t}(C-u)=\liminf _{t \downarrow 0} \frac{1}{t}(C-u),
$$

where the first limit set if defined for an arbitrary set $C$ is called the contingent cone of $C$ at $u$ and the second limit set which is lying between the contingent cone and the Clarke tangent cone [5] is referred to as the derivative cone of $C$ at $u$. These cones are known to coincide when $C$ is a convex set [5, 22.4$]$.

Example 2.7. Let $C$ be a closed convex set in $E$. Let $\sigma_{C}$ be the support function and $\delta_{C}$ be the indicator function of $C$. One has

(a) $\left(\partial \sigma_{C}\right)(0)=C$ and $\left(\sigma_{C}\right)_{0, u}^{\prime \prime}=\delta_{N_{C}(u)}$ for all $u \in C$.

(b) $\left(\delta_{C}\right)_{u, 0}^{\prime \prime}=\delta_{T_{C}(u)}$ for all $u \in C$.

(c) Let $p=\|\cdot\|$ be the norm function on $E$. Then $p_{0, u}^{\prime \prime}=\delta_{N_{B^{*}}(u)}$ for all $u \in B^{*}$, where $B^{*}$ denotes the closed unit ball in $E$.

Proof. (a) Let $\sigma=\sigma_{C}$. One has

$$
u \in \partial \sigma(0) \Leftrightarrow 0 \in \partial \sigma^{*}(u)=\partial \delta_{C}(u) \Leftrightarrow u \in C .
$$

Let $\varphi_{t}$ be the second-order difference quotient function of $\sigma$ at 0 relative to $u \in C$. Then

$$
\varphi_{t}(\xi)=\frac{2}{t}[\sigma(\xi)-\langle u, \xi\rangle]
$$

Let $N=N_{C}(u)=\{\xi:\langle v, \xi\rangle \leq\langle u, \xi\rangle, \forall v \in C\}=\{\xi: \sigma(\xi)=\langle u, \xi\rangle\}$ and $\varphi=\delta_{N}$. It is obvious that (1.9) and (1.10) are satisfied for every $\xi \in N$. To verify $\varphi_{t} \stackrel{M}{\rightarrow} \varphi$, we only need to show (1.9) holds for $\xi \notin N$ or

$$
\forall \xi \notin N, \quad \forall t_{n} \downarrow 0, \quad \forall \xi_{n} \stackrel{w}{\rightarrow} \xi \quad \liminf _{n} \varphi_{t_{n}}\left(\xi_{n}\right)=\infty .
$$

For $\xi \notin N$, let $\alpha=\frac{1}{3}(\sigma(\xi)-\langle u, \xi\rangle)>0$. Since $\lim \left\langle u, \xi_{n}\right\rangle=\langle u, \xi\rangle$ and $\liminf \sigma\left(\xi_{n}\right) \geq \sigma(\xi) \quad$ (weak lower-semicontinuity of $\sigma$ ), one has $\left\langle u, \xi_{n}\right\rangle<$ $\langle u, \xi\rangle+\alpha$ and $\sigma\left(\xi_{n}\right)>\sigma(\xi)-\alpha$ for all $n$ large enough. Thus $\varphi_{t_{n}}\left(\xi_{n}\right)=$ $\frac{2}{t_{n}}\left[\sigma\left(\xi_{n}\right)-\left\langle u, \xi_{n}\right\rangle\right]>\frac{2}{t_{n}}[\sigma(\xi)-\langle u, \xi\rangle-2 \alpha]=\left(2 / t_{n}\right) \alpha$ for $n$ large. Hence $(2.7)$ holds.

(b) One has by part (a) and Theorem 2.5,

$$
\frac{1}{2}\left(\delta_{C}\right)_{u, 0}^{\prime \prime}=\left(\frac{1}{2} \sigma_{0, u}^{\prime \prime}\right)^{*}=\delta_{N}^{*}=\delta_{T_{C}(u)} .
$$

(c) Note that the norm function $p$ in $E$ is the support function of the closed unit ball $B^{*}$ in $E^{*}$, so part (c) follows from (a).

Definition 2.8. A closed convex set $C$ in $E$ is called polyhedric in the sense of Haraux in [8] if, for every $u \in C$ and for every $z \in N_{C}(u)$, one has

$$
\begin{aligned}
& \left\{\eta: \eta \in T_{C}(u) \text { and }\langle z, \eta\rangle=0\right\} \\
& \quad=\operatorname{cl}\{\eta:\langle z, \eta\rangle=0 \text { and } \exists \lambda>0, u+\lambda \eta \in C\} .
\end{aligned}
$$

In particular, $C$ is polyhedric when (compare with (2.5))

$$
T_{C}(u)=\{\eta: \exists \lambda>0, u+\lambda \eta \in C\}, \quad \forall u \in C .
$$

If $E$ is a Hilbert space and $P$ is the projection mapping on $C$, then $P(x)=$ $u \Leftrightarrow u \in C$ and $x-u \in N_{C}(u)$ (Corollary 4.4). Thus the above definition is the same as that given in $[8,21]$. 
Lemma 2.9. Let $C$ be a closed convex set in $E$. The following are equivalent.

(a) $C$ is polyhedric.

(b) For every $u \in C$, for every $z \in N_{C}(u)$, let $\mathscr{N}$ be the tangent cone to $N_{C}(u)$ at $z$. One has

$$
\mathscr{N}=\{\eta \in E:\langle z, \eta\rangle=0 \text { and } \exists \lambda>0, u+\lambda \eta \in C\}^{\circ} .
$$

Proof. Note that $T_{K}(z)=\operatorname{cl}(K+[z])$ for any convex cone $K$ and $z \in K$, where $[z]$ denotes the linear space generated by $z$. Hence

$$
\begin{aligned}
\mathscr{N}^{\circ} & =\left(N_{C}(u)+[z]\right)^{\circ}=T_{C}(u) \cap[z]^{\circ} \\
& =\left\{\eta: \eta \in T_{C}(u) \text { and }\langle z, \eta\rangle=0\right\} .
\end{aligned}
$$

Therefore by taking polarity, one can see that (2.8) and (2.10) are equivalent.

Example 2.10. Let $C$ be a closed convex set which is polyhedric. Then

(a) The support function $\sigma_{C}$ is essentially twice epi-differentiable.

(b) The indicator function $\delta_{C}$ is twice epi-differentiable. For $z \in E, u \in$ $E^{*}$, one has

$$
\begin{aligned}
u \in \partial \sigma_{C}(z) & \Leftrightarrow u \in C \text { and } z \in N_{C}(u) \\
& \Leftrightarrow u \in C \text { and } \sigma_{C}(z)=\langle u, z\rangle, \\
\left(\sigma_{C}\right)_{z, u}^{\prime \prime}=\delta_{\mathscr{N}}, & \text { where } \mathscr{N}=\operatorname{cl}\left(N_{C}(u)+[z]\right), \\
\left(\delta_{C}\right)_{u, z}^{\prime \prime}=\delta_{\mathscr{T}}, & \text { where } \mathscr{T}=\left\{\eta \in T_{C}(u):\langle z, \eta\rangle=0\right\} .
\end{aligned}
$$

(In (2.12), [z] denotes the linear space spanned by $z$.)

Proof. To show (a) and (b), it suffices to verify (2.11)-(2.13). The equivalences in (2.11) are well-known facts in convex analysis. Let $\varphi_{t}$ be the second-order difference quotient of $\sigma=\sigma_{C}$ at $z$ relative to $u$. One has

$$
\begin{aligned}
\varphi_{t}(\xi) & =\frac{2}{t^{2}}[\sigma(z+t \xi)-\sigma(z)-t\langle u, \xi\rangle] \\
& =\frac{2}{t^{2}}[\sigma(z+t \xi)-\langle u, z+t \xi\rangle] \\
& =\frac{2}{t^{2}} \sup _{v \in C}[\langle z+t \xi, v-u\rangle], \quad \xi \in E .
\end{aligned}
$$

Let $\mathcal{N}$ be the set in (2.12). Then $\mathcal{N}$ is the tangent cone to $N_{C}(u)$ at $z$ as claimed in the proof of Lemma 2.9:

$$
\mathscr{N}=\underset{t \downarrow 0}{\lim \sup } \frac{1}{t}\left(N_{C}(u)-z\right)=\liminf _{t \downarrow 0} \frac{1}{t}\left(N_{C}(u)-z\right) .
$$

In view of (1.9) and (1.10), (2.12) is established if the following are shown:

$$
\begin{array}{llll}
\forall \xi \in \mathscr{N} & \forall t_{n} \downarrow 0, & \exists \xi_{n} \stackrel{s}{\rightarrow} \xi, & \lim \sup _{n} \varphi_{t_{n}} \leq 0, \\
\forall \xi \notin \mathscr{N} & \forall_{t_{n}} \downarrow 0, \quad \forall \xi_{n} \stackrel{w}{\rightarrow} \xi, & \lim _{n} \inf \varphi_{t_{n}}=\infty
\end{array}
$$

Let $\xi \in \mathscr{N}$ and $t_{n} \downarrow 0$. From (2.15), there exists a sequence $\xi_{n} \stackrel{s}{\rightarrow} \xi, z+t_{n} \xi_{n} \in$ $N_{C}(u)$. Then $\left\langle z+t_{n} \xi_{n}, v-u\right\rangle \leq 0$ for all $v \in C$. Thus $\varphi_{t}\left(\xi_{n}\right) \leq 0$ by (2.14). Hence (2.16) holds. 
Let $\xi \notin \mathscr{N}$. Then by Lemma 2.9(b), there exists an $\eta$ such that $\alpha:=$ $\langle\xi, \eta\rangle>0,\langle z, \eta\rangle=0$, and $u+\lambda \eta \in C$ for some $\lambda>0$. By (2.14) one has

$$
\varphi_{t}\left(\xi^{\prime}\right) \geq \frac{2}{t^{2}}\left\langle z+t \xi^{\prime}, \lambda \eta\right\rangle=\frac{2 \lambda}{t}\left\langle\xi^{\prime} \eta\right\rangle, \quad \xi^{\prime} \in E .
$$

For any $\xi_{n} \stackrel{w}{\rightarrow} \xi$, we have $\lim \left\langle\xi_{n}, \eta\right\rangle=\langle\xi, \eta\rangle=\alpha$. Thus

$$
\varphi_{t_{n}}\left(\xi_{n}\right) \geq \frac{2 \lambda}{t_{n}} \frac{\alpha}{2}=\frac{\lambda \alpha}{t_{n}} \quad \text { for } n \text { sufficiently large. }
$$

This shows (2.17). We have shown (2.12). By Theorem 2.5 and the polarity relationship between the two sets $\mathscr{N}$ and $\mathscr{T}$, we also obtain (2.13). The proof is complete.

\section{Proto-differentiation}

Definition 3.1. Given a multifunction $\Gamma: E \rightrightarrows F$, a point $x \in E$ with $\Gamma(x) \neq$ $\varnothing$ and a point $z \in \Gamma(x)$, consider the difference quotient multifunctions

$$
\left(\Delta_{t} \Gamma\right)_{x, z}(\xi):=\frac{1}{t}\{\Gamma(x+t \xi)-z\}, \quad \xi \in E(t>0) .
$$

If the graphs of $\left(\Delta_{t} \Gamma\right)_{x, z}$ as a family of subsets of $E \times F$ strongly converge (as $t \downarrow 0$ ) to another subset of $E \times F$ (in the sense of (1.15) or (1.17)), then $\Gamma$ is said to be proto-differentiable at $x$ relative to $z$, and the limit set is the graph of another multifunction $\Gamma_{x, z}^{\prime}: E \rightrightarrows F$ which will be called the proto-derivative of $\Gamma$ at $x$ relative to $z$. We write $\Gamma_{x, z}^{\prime}=\operatorname{G}_{-1 i m} \lim _{t \downarrow}\left(\Delta_{t} \Gamma\right)_{x, z}$. In terms of sequences,

$$
\Gamma_{x, z}^{\prime}=\underset{n \rightarrow \infty}{\mathrm{G}-\lim _{n}}\left(\Delta_{t_{n}} \Gamma\right)_{x, z}, \quad \forall t_{n} \downarrow 0 .
$$

Note that the graph of $\left(\Delta_{t} \Gamma\right)_{x, z}$ is the set

$$
\operatorname{gph}\left(\Delta_{t} \Gamma\right)_{x, z}=\frac{1}{t}[\operatorname{gph} \Gamma-(x, z)],
$$

so that $\Gamma$ is proto-differentiable at $x$ relative to $z$ if and only if the contingent cone of $\operatorname{gph} \Gamma$ at $(x, z)$, which is

$$
K_{\Gamma}(x, z)=\limsup _{t \downarrow 0} \frac{1}{t}[\operatorname{gph} \Gamma-(x, z)],
$$

coincides with the derivative cone

$$
D_{\Gamma}(x, z)=\liminf _{t \downarrow 0} \frac{1}{t}[\operatorname{gph} \Gamma-(x, z)] .
$$

The common cone is the graph of the proto-derivative.

$\Gamma$ is said to be proto-differentiable at $x$ if $\Gamma(x) \neq \varnothing$, and for every $z \in$ $\Gamma(x), \Gamma$ is proto-differentiable at $x$ relative to $z$. One can see that the protodifferentiability is a local property.

Proposition 3.2. Let $\Gamma: E \rightrightarrows F$ be proto-differentiable at $x$ relative to $z \in$ $\Gamma(x)$. Then the proto-derivative $\Gamma_{x, z}^{\prime}: E \rightrightarrows F$ has closed graph and satisfies

$$
0 \in \Gamma_{x, z}^{\prime}(0) \text { and } \Gamma_{x, z}^{\prime}(\lambda \xi)=\lambda \Gamma_{x, z}^{\prime}(\xi)
$$

for all $\xi \in E$ and $\lambda>0$. 
If $F=E^{*}$ and $\Gamma$ is a monotone operator, then for every $\xi \in \operatorname{dom} \Gamma_{x, z}^{\prime}$ and for every $\eta_{1}, \eta_{2} \in \Gamma_{x, z}^{\prime}(\xi)$, one has $\left\langle\eta_{1}, \xi\right\rangle=\left\langle\eta_{2}, \xi\right\rangle$.

Proof. The verification of the first part is direct as in the finite-dimensional case (see [16, Proposition 2.3]).

Suppose the second part were false, there would exist $\eta_{1}, \eta_{2} \in \Gamma_{x, z}^{\prime}(\xi)$ such that $\left\langle\eta_{1}, \xi\right\rangle<\left\langle\eta_{2}, \xi\right\rangle$. Let $\lambda>1$ such that

$$
\lambda\left\langle\eta_{1}, \xi\right\rangle<\left\langle\eta_{2}, \xi\right\rangle \text {. }
$$

By homogeneity of $\Gamma_{x, z}^{\prime}$, one has $\lambda \eta_{1} \in \Gamma_{x, z}^{\prime}(\lambda \xi)$. Since $\Gamma$ is monotone, its proto-derivative is also monotone. Hence

$$
\left\langle\lambda \eta_{1}-\eta_{2}, \lambda \xi-\xi\right\rangle \geq 0 \text { or }(\lambda-1)\left\langle\lambda \eta_{1}-\eta_{2}, \xi\right\rangle \geq 0 .
$$

This implies that $\left\langle\lambda \eta_{1}-\eta_{2}, \xi\right\rangle \geq 0$. Thus $\lambda\left\langle\eta_{1}, \xi\right\rangle \geq\left\langle\eta_{2}, \xi\right\rangle$ which contradicts (3.4). The proof is complete.

Proposition 3.3. Suppose $A=\left(A_{1}, A_{2}\right): E_{1} \times F_{1} \rightarrow E_{2} \times F_{2}$ is a linear isomorphism and $\Gamma: E_{1} \rightrightarrows F_{1}$ is a multifunction. Let $A \Gamma$ be the multifunction: $E_{2} \rightrightarrows F_{2}$ defined by $\operatorname{gph}(A \Gamma)=A[\operatorname{gph} \Gamma]$. Assume $\Gamma(x) \neq \varnothing$, and let $z \in \Gamma(x)$. Then $\Gamma$ is proto-differentiable at $x$ relative to $z$ if and only if $A \Gamma$ is proto-differentiable at $A_{1}(x, z)$ relative to $A_{2}(x, z)$. One has $A \Gamma_{x, z}^{\prime}=$ $(A \Gamma)_{A_{1}(x, z), A_{2}(x, z)}^{\prime}$.

Proof. Let $\Sigma=A \Gamma$. Then

$$
A\left[\operatorname{gph}\left(\Delta_{t} \Gamma\right)_{x . z}\right]=\operatorname{gph}\left(\Delta_{t} \Sigma\right)_{A_{1}(x, z), A_{2}(x, z)} .
$$

Observe that for any family sets $D_{t} \subset E_{1} \times F_{1} \quad(t>0), D_{t} \stackrel{s}{\rightarrow} D$ if and only if $A\left[D_{t}\right] \stackrel{s}{\rightarrow} A[D]$, so the proposition follows.

Corollary 3.4. Let $\Gamma: E \rightrightarrows F$. Suppose $\Gamma(x) \neq \varnothing$ and $z \in \Gamma(x)$. Then $\Gamma$ is proto-differentiable at $x$ relative to $z$ if and only if $\Gamma^{-1}$ is proto-differentiable at $z$ relative to $x$. One has $\left(\Gamma^{-1}\right)_{z, x}^{\prime}=\left(\Gamma_{x, z}^{\prime}\right)^{-1}$.

(The inverse $\Gamma^{-1}$ of $\Gamma$ is defined by $x \in \Gamma^{-1}(z) \Leftrightarrow z \in \Gamma(x)$.)

Proof. Apply Proposition 3.3 with $A: E \times F \rightarrow F \times E$ defined by $A(x, z)=$ $(z, x)$.

The next several results connect proto-differentiability with classical differentiability when the given multifunction happens to be single-valued (a mapping). It should be noted here that even if a single-valued mapping $\Gamma$ is continuous and proto-differentiable at $x$, its proto-derivative might not be single-valued as seen by the following.

Example. Let $\Gamma(x)=\sqrt{|x|}: \mathbf{R} \rightarrow \mathbf{R}$. Let $D_{t}$ be the first-order difference quotient function of $\Gamma$ at 0 relative to $0=\Gamma(0)$. Then $D_{t}(\xi)=\sqrt{|\xi| / t}$. Thus G- $\lim _{t \downarrow 0} D_{t}=D$ exists with

$$
D(\xi)= \begin{cases}{[0, \infty)} & \text { if } \xi=0, \\ \varnothing & \text { if } \xi \neq 0,\end{cases}
$$

and $D$ is the proto-derivative of $\Gamma$ at 0 (relative to 0 ). 
Proposition 3.5. Let $\Gamma: E \rightrightarrows F$ be single valued in a neighborhood $U$ of $x$. Suppose that $\Gamma$ has one-sided directional derivative at $x$ relative to every $\xi \in E$,

$$
D(\xi):=\lim _{t \downarrow 0} \frac{\Gamma(x+t \xi)-\Gamma(x)}{t}, \quad \xi \in E .
$$

Suppose also that the convergence in (3.5) is uniform for $\xi$ in a compact set and that $D$ is a continuous mapping. Then $\Gamma$ is proto-differentiable at $x$ and $\Gamma_{x, \Gamma(x)}^{\prime}=D$.

Let $\Gamma$ be Lipschitzian in $U$ with constant $k$. Then the existence of the limits in (3.5) is equivalent to $\Gamma$ being proto-differentiable at $x$ relative to $\Gamma(x)$ with $\operatorname{dom} \Gamma_{x, \Gamma(x)}^{\prime}=E$ (this condition is superfluous if $F$ is finite-dimensional). In this case D given in (3.5) is Lipschitzian with the same constant $k$ and the convergence is uniform for $\xi$ in a compact set.

Proof. By the uniform convergence in (3.5), one can write

$$
\Gamma(x+t \xi)-\Gamma(x)=t D(\xi)+o(t, \xi), \quad t>0, \xi \in E,
$$

where $\lim _{t \downarrow 0} o(t, \xi) / t=0$ with the convergence being uniform for $\xi$ in a compact set.

A sufficient condition for the proto-differentiability of $\Gamma$ at $x$ with $\Gamma_{x, \Gamma(x)}^{\prime}=$ $D$ is that for every sequence $t_{n} \downarrow 0$, for every sequence $\xi_{n} \stackrel{s}{\rightarrow} \xi$,

$$
\lim _{n} \frac{\Gamma\left(x+t_{n} \xi_{n}\right)-\Gamma(x)}{t_{n}}=D(\xi) \text {. }
$$

By (3.6), this holds when

$$
\lim _{n} \frac{o\left(t_{n}, \xi_{n}\right)}{t_{n}}=0 \text { and } \lim _{n} D\left(\xi_{n}\right)=D(\xi) .
$$

These conditions are satisfied because $\left\{\xi_{n}, \xi\right\}$ is a compact set and $D$ is a continuous mapping. The first part of the proposition is proved.

For the second part, let $\Gamma$ be Lipschitzian in $U$ with constant $k$. Let $D_{t}$ be the first-order difference quotient of $\Gamma$ at $x$ relative to $\Gamma(x)$. Then $D_{t}$ is Lipschitzian with the same constant.

Let $t_{n} \downarrow 0, D_{n}=D_{t_{n}}$ and suppose that $\Gamma$ is proto-differentiable at $x$ with $\Gamma_{x, \Gamma(x)}^{\prime}=D$. We wish to show that $D$ is given in (3.5). Let $\xi \in E$ and $\eta \in D(\xi)$ $(\operatorname{dom} D=E)$. Since $\operatorname{gph} D \subset \mathrm{s}-\lim \inf \left(\operatorname{gph} D_{t}\right)$, there exists a sequence $\xi_{n} \stackrel{s}{\rightarrow} \xi$ such that $D_{n}\left(\xi_{n}\right) \stackrel{s}{\rightarrow} \eta$. One has for any $\xi_{n}^{\prime} \stackrel{s}{\rightarrow} \xi$ that

$\left\|D_{n}\left(\xi_{n}^{\prime}\right)-\eta\right\| \leq\left\|D_{n}\left(\xi_{n}^{\prime}\right)-D_{n}\left(\xi_{n}\right)\right\|+\left\|D_{n}\left(\xi_{n}\right)-\eta\right\| \leq k\left\|\xi_{n}^{\prime}-\xi_{n}\right\|+\left\|D_{n}\left(\xi_{n}\right)-\eta\right\|$

so that $D_{n}\left(\xi_{n}^{\prime}\right) \stackrel{s}{\rightarrow} \eta$. This argument shows that $D(\xi)$ is a singleton and $D(\xi)=$ $\lim D_{n}\left(\xi_{n}\right)$. This is true for every sequence $t_{n} \downarrow 0$, hence (3.5) holds.

The condition $\operatorname{dom} D=E$ above is automatically satisfied when $F$ is finitedimensional. In fact, let $\xi \in E$ and consider $\left\{D_{n}(\xi)\right\}$. This sequence is bounded by Lipschitzian property and by $D_{n}(0)=0$. Therefore it has a limit point, say $\eta$. Thus $(\xi, \eta) \in \lim \sup \left[\operatorname{gph} D_{n}\right] \subset \operatorname{gph} D$, hence $\xi \in \operatorname{dom} D$.

The Lipschitzian property of $D$ follows from that of $D_{n}$. The uniform convergence for $\xi$ in a compact set in (3.5) can be seen by a standard compactness argument using the Lipschitzian property of $D_{t}$ and $D$. 
Corollary 3.6. If $\Gamma: E \rightrightarrows F$ is Hadamard differentiable at $x$, then it is protodifferentiable at $x$ and $\Gamma_{x, \Gamma(x)}^{\prime}=D \Gamma(x)$, the Hadamard derivative of $\Gamma$ at $x$. The converse is true when $\Gamma$ is Lipschitzian in a neighborhood of $x$ and $\Gamma_{x, \Gamma(x)}^{\prime}$ is a continuous linear mapping.

Proposition 3.7. Let $\Gamma: E \rightrightarrows \mathbf{R}^{k}$ be single-valued in a neighborhood $U$ of $x$. Suppose $\Gamma$ is continuous and proto-differentiable at $x$ with its proto-derivative $D=\Gamma_{x, \Gamma(x)}^{\prime}$ being single-valued everywhere. Then the one-sided directional derivative of $\Gamma$ at $x$ relative to every $\xi \in E$ exists and

$$
D(\xi)=\lim _{t \downarrow 0} \frac{\Gamma(x+t \xi)-\Gamma(x)}{t} .
$$

If in addition $D$ is continuous, then the convergence in (3.8) is uniform for $\xi$ in a compact set.

Proof. Let

$$
D_{t}(\xi)=\frac{\Gamma(x+t \xi)-\Gamma(x)}{t}, \quad \xi \in E, t>0 .
$$

We wish to show that for every bounded set $B$, there exists some $\delta>0$ such that $\left\{D_{t}(\xi): \xi \in B, 0<t<\delta\right\}$ is bounded. If this were false, there would exist a sequence $s_{n} \rightarrow 0$, a sequence $\xi_{n}^{\prime} \in B$ such that $\alpha_{n}:=\left\|D_{S_{n}}\left(\xi_{n}^{\prime}\right)\right\| \rightarrow \infty$ and $D_{s_{n}}\left(\xi_{n}^{\prime}\right) / \alpha_{n} \rightarrow$ some $\eta$ with $\|\eta\|=1$. Let $t_{n}=\alpha_{n} s_{n}, \xi_{n}=\left(\xi_{n}^{\prime} / \alpha_{n}\right)$. Then $\xi_{n} \rightarrow 0, t_{n} \rightarrow 0$ (since $\Gamma$ is continuous at $x$ ) and $D_{t_{n}}\left(\xi_{n}\right)=D_{S_{n}}\left(\xi_{n}^{\prime}\right) / \alpha_{n} \rightarrow \eta$. This shows that $(0, \eta) \in \lim \sup \left[\operatorname{gph} D_{t}\right] \subset \operatorname{gph} D$. Hence $\eta \in D(0)=\{0\}$, a contradiction. Therefore $\left\{D_{t}(\xi): 0<t<\delta, \xi \in B\right\}$ is bounded as claimed.

Let $\xi \in E, \xi_{n} \rightarrow \xi$, and $t_{n} \downarrow 0$. By the above result, $\left\{D_{t_{n}}\left(\xi_{n}\right)\right\}$ is bounded, hence it has limit points in $\mathbf{R}^{k}$. For any limit point $\eta$ of $\left\{D_{t_{n}}\left(\xi_{n}\right)\right\}$, one has $(\xi, \eta)\} \in \lim \sup \left[\operatorname{gph} D_{t}\right]=\operatorname{gph} D$, hence $\eta \in D(\xi)$. The single-valuedness of $D$ implies that $\left\{D_{t_{n}}\left(\xi_{n}\right)\right\}$ has a unique limit point $D(\xi)$. Therefore $D(\xi)=$ $\lim _{n} D_{t_{n}}\left(\xi_{n}\right)$. This is true for any sequences $\xi_{n} \rightarrow \xi, t_{n} \downarrow 0$, hence

$$
D(\xi)=\lim _{\substack{t \perp 0 \\ \xi^{\prime} \rightarrow \xi}} D_{t}\left(\xi^{\prime}\right)
$$

In particular, (3.8) holds.

Suppose $D$ is continuous. Let $C$ be a compact set. Let $\varepsilon>0$. Then by (3.9), the continuity of $D$, and the compactness of $C$, there exists a $\delta>0$ such that $\left|D_{t}(\xi)-D(\xi)\right|<\varepsilon$ for $0<t<\delta, \xi \in C$. This shows the uniform convergence in (3.8).

Corollary 3.8 [16, Theorem 3.5]. Let $\Gamma: E \rightarrow \mathbf{R}^{k}$ be a mapping which is continuous at $x \in E$. Then $\Gamma$ is Hadamard differentiable at $x$ if and only if $\Gamma$ is proto-differentiable at $x$ with $\Gamma_{x, \Gamma(x)}^{\prime}$ being a continuous linear mapping.

The next theorem connects the epi-differentiability of a convex function with the proto-differentiability of its subdifferential.

Theorem 3.9. Let $f: E \rightarrow \overline{\mathbf{R}}$ be a closed proper convex function. Let $x \in E$ and assume that $f(x)$ is finite. Then the following are equivalent:

(a) $f$ is twice epi-differentiable at $x$ relative to $z$.

(b) $z \in \partial f(x)$ and $\partial f$ is proto-differentiable at $x$ relative to $z$ with the proto-derivative being a maximal monotone operator. 
One then has

$$
\partial\left(\frac{1}{2} f_{x, z}^{\prime \prime}\right)(\xi)=(\partial f)_{x, z}^{\prime}(\xi), \quad \xi \in E .
$$

Proof. First, we state Attouch's theorem (cf. [1, Theorem 3.66]):

Attouch's theorem. Let $\left(\varphi_{n}\right), \varphi$ be a sequence of closed proper convex functions. Then $\varphi=\mathrm{M}-\lim \varphi_{n}$ if and only if the following conditions hold:

(i) $\partial \varphi=\mathrm{G}-\lim \left(\partial \varphi_{n}\right)$.

(ii) $\exists(\xi, \eta) \in \operatorname{gph} \partial \varphi, \exists\left(\xi_{n}, \eta_{n}\right) \in \partial \varphi_{n}$ such that $\left(\xi_{n}, \eta_{n}\right) \rightarrow(\xi, \eta)$ and $\varphi_{n}\left(\xi_{n}\right) \rightarrow \varphi(\xi)$.

Now, for any $t>0$, let

$$
\varphi_{t}(\xi)=\left(\Delta_{t}^{(2)} f\right)_{x, z}(\xi)=\frac{2}{t^{2}}\{f(x+t \xi)-f(x)-t\langle z, \xi\rangle\}, \quad \xi \in E .
$$

Then

$$
\partial \frac{1}{2} \varphi_{t}(\xi)=\frac{1}{t}\{\partial f(x+t \xi)-z\}=\Delta_{t}(\partial f)_{x, z}(\xi),
$$

which is the difference quotient for the multifunction $\partial f$. We have

$$
\begin{gathered}
\frac{1}{2} \varphi_{t}(0)=0, \\
0 \in \partial \frac{1}{2} \varphi_{t}(0) \Leftrightarrow z \in \partial f(x) .
\end{gathered}
$$

Suppose (a) in the theorem holds. Then $z \in \partial f(x)$ by Proposition 2.3. If $\varphi=\frac{1}{2} f_{x, z}^{\prime \prime}$, then for every sequence $t_{n} \downarrow 0$, the sequence $\varphi_{n} \equiv \frac{1}{2} \varphi_{t_{n}}$ Mosco converges to $\varphi$ (as $n \rightarrow \infty)$, so by Attouch's theorem, (i) holds, hence (3.11) implies that $\Delta_{t_{n}}(\partial f)_{x, z}$ graphically converges to $\partial \varphi$. We conclude that the G-limit of $\Delta_{t}(\partial f)_{x, z}$ exists and

$$
\partial \frac{1}{2} f_{x, z}^{\prime \prime}=\partial \varphi=\mathrm{G}-\lim \Delta_{t}(\partial f)_{x, z}=(\partial f)_{x, z}^{\prime} .
$$

This proves (b) and (3.10).

Conversely, suppose (b) of the theorem holds, i.e. $z \in \partial f(x)$ and there is a maximal monotone operator $\Phi: E \rightrightarrows E^{*}$ such that

$$
\Phi=\mathrm{G}-\lim _{n} \Delta_{t_{n}}(\partial f)_{x, z}, \quad \forall t_{n} \downarrow 0 .
$$

Since the class of subdifferentials of closed proper convex functions is closed under graphical convergence in the class of maximal monotone operators ( $\mathrm{cf}$. [1, Corollary 3.6]), one has $\Phi=\partial \varphi$ for some closed proper convex function $\varphi$. By (3.11), one further has

$$
\partial \varphi=\mathrm{G}-\lim \partial \frac{1}{2} \varphi_{t_{n}},
$$

and then by (3.13), $0 \in \partial \frac{1}{2} \varphi_{t_{n}}(0)=\Delta_{t_{n}}(\partial f)_{x, z}(0)$. The graphical convergence of $\Delta_{t_{n}}(\partial f)$ to $\Phi$ gives that $0 \in \Phi(0)=\partial \varphi(0)$. In particular, $\varphi(0)$ is finite. By adding a constant, we can assume that $\varphi(0)=0$. From (3.12), we see that (ii) of Attouch's theorem holds for $\frac{1}{2} \varphi_{t_{n}}$ and $\varphi$ by taking $\xi_{n}=0, \eta_{n}=0$. Combining this with (3.14), we conclude from Attouch's theorem that $\varphi=\mathrm{M}-\lim \frac{1}{2} \varphi_{t_{n}}$. This is true for any sequence $t_{n} \downarrow 0$, so the Mosco epi-limit of the second-order difference quotient functions $\frac{1}{2} \varphi_{t}$ exists and the limit is $\varphi$, with $\varphi(0)=0$. This proves $(\mathrm{a})$. 


\section{Some calculus}

Proposition 4.1. Let $f: E \rightarrow \overline{\mathbf{R}}$ be a (Fréchet) differentiable convex function in a neighborhood $U$ of $x \in E$. If $f$ is twice Fréchet differentiable at $x$, then $f$ is twice epi-differentiable at $x$ with its epi-derivative $f_{x, D f(x)}^{\prime \prime}$ being a continuous quadratic form. One has

$$
f_{x, D f(x)}^{\prime \prime}(\xi)=\left\langle D^{2} f(x) \xi, \xi\right\rangle, \quad \xi \in E .
$$

The converse is true when $E$ is finite-dimensional.

Proof. Suppose $f$ is twice Fréchet differentiable at $x$. Then $D f$ is Fréchet differentiable at $x$. Thus by Corollary 3.6, $D f$ is proto-differentiable at $x$ and $(D f)_{x, D f(x)}^{\prime}(\xi)=D^{2} f(x) \xi \quad(\xi \in E)$. Because $(D f)_{x, D f(x)}^{\prime}$ is the graphical limit of monotone operators, it is monotone. Moreover, it is single-valued and continuous, hence is maximal. By Theorem 3.9, $f$ is twice epi-differentiable at $x$ and

$$
\partial\left(\frac{1}{2} f_{x, D f(x)}^{\prime \prime}\right)(\xi)=(D f)_{x, D f(x)}^{\prime}(\xi)=D^{2} f(x) \xi, \quad \xi \in E .
$$

Since $f_{x, D f(x)}^{\prime \prime}(0)=0$, this subdifferential equation yields

$$
f_{x, D f(x)}^{\prime \prime}(\xi)=\left\langle D^{2} f(x) \xi, \xi\right\rangle, \quad \xi \in E .
$$

Thus the first part and (4.1) hold.

Conversely, suppose $E=\mathbf{R}^{k}$ and $f$ is twice epi-differentiable at $x$ with $f_{x, D f(x)}^{\prime \prime}(\xi)=\xi \cdot H \xi \quad(\xi \in E)$, where $H$ is a (positively semidefinite) matrix. By Theorem 3.9, $D f$ is proto-differentiable at $x$ and $(D f)_{x, D f(x)}^{\prime}(\xi)=H \xi \quad(\xi \in$ $E$ ) which is a continuous linear operator on $E$. Moreover, the (once) Fréchet differentiability of $f$ in a (convex) neighborhood of $x$ necessarily implies that $D f$ is continuous at $x$ (cf. [12, Proposition 2.8]), therefore by Corollary 3.8, $D f$ is Fréchet differentiable at $x$, i.e. $f$ is twice Fréchet differentiable at $x$.

Proposition 4.2. Suppose $f$ and $g$ are closed proper convex functions on $E$ and $f$ is twice Fréchet differentiable at $x \in E$. Let $h=f+g$. Then

$$
z=D f(x)+u \in \partial h(x) \Leftrightarrow u \in \partial g(x) .
$$

Function $g$ is twice epi-differentiable at $x$ relative to $u$ if and only if $h$ is twice epi-differentiable at $x$ relative to $z$, and

$$
h_{x, z}^{\prime \prime}(\xi)=\left\langle D^{2} f(x) \xi, \xi\right\rangle+g_{x, u}^{\prime \prime}(\xi), \quad \xi \in E .
$$

Proof. The first statement is a consequence of the well-known fact in convex analysis:

$$
\partial h(y)=\partial f(y)+\partial g(y), \quad y \in E,
$$

if both functions $f$ and $g$ are finite at some point at which at least one is continuous.

To prove the second part of the proposition, let $\Phi_{t}, \Gamma_{t}, \Delta_{t}$ be the difference quotients of $\partial h$ (at $x$ relative to $z$ ), $D f($ at $x$ relative to $D f(x))$ and $\partial g$ (at $x$ relative to $u$ ) respectively. One has

$$
\Phi_{t}(\xi)=\Gamma_{t}(\xi)+\Delta_{t}(\xi), \quad \xi \in E .
$$


Since $D f$ is Fréchet differentiable, one has (see Propositions 3.5 and (3.7))

$$
\lim _{n} \Gamma_{t_{n}}\left(\xi_{n}\right)=D^{2} f(x) \xi, \quad \forall \xi \in E, \xi_{n} \rightarrow \xi, t_{n} \downarrow 0 .
$$

Suppose now that $g$ is twice epi-differentiable at $x$ relative to $u$. We wish to show that

$$
\underset{t \downarrow 0}{\mathrm{G}-\lim } \Phi_{t}=D^{2} f(x)+(\partial g)_{x, u}^{\prime}=\frac{1}{2} \partial\left(f_{x, D f(x)}^{\prime \prime}+g_{x, u}^{\prime \prime}\right),
$$

where the second equality comes from Theorem 3.9 and the twice epi-differentiability of $g$. Since the right-most is a maximal monotone operator, it suffices to show that $\operatorname{gph}\left[D^{2} f(x)+(\partial g)_{x, u}^{\prime}\right] \subset \liminf _{n}\left[\operatorname{gph} \Phi_{t_{n}}\right]$ for every sequence $t_{n} \downarrow 0$, i.e.,

$$
\begin{aligned}
& \left.D^{2} f(x) \xi+\eta \in \underset{n}{\liminf [g p h} \Phi_{t_{n}}\right](\xi), \\
& \forall \xi \in \operatorname{dom}(\partial g)_{x, u}^{\prime}, \quad \forall \eta \in(\partial g)_{x, u}^{\prime}(\xi), \quad \forall t_{n} \downarrow 0 .
\end{aligned}
$$

To show (4.7), it is enough to produce sequences $\xi_{n} \rightarrow \xi, \omega_{n} \in \Phi_{t_{n}}\left(\xi_{n}\right)$ such that $\omega_{n} \rightarrow D^{2} f(x) \xi+\eta$. Since $\Delta_{t}$ graphically converges to $(\partial g)_{x, u}^{\prime}$ (Theorem 3.9), one can pick sequences $\xi_{n} \rightarrow \xi$ and $\eta_{n} \in \Delta_{t_{n}}\left(\xi_{n}\right)$ such that $\eta_{n} \rightarrow \eta$. By (4.4) and (4.5), we can choose $\omega_{n}=\Gamma_{t_{n}}\left(\xi_{n}\right)+\eta_{n}$. Thus (4.7) holds.

We have therefore shown (4.6). Thus $\partial h$ is proto-differentiable at $x$ relative to $z$ with $(\partial h)_{x, z}^{\prime}=\frac{1}{2} \partial\left(f_{x, D f(x)}^{\prime \prime}+g_{x, u}^{\prime \prime}\right)$. By Theorem 3.9, $h$ is twice epi-differentiable at $x$ relative to $z$ and $\partial\left(h_{x, z}^{\prime \prime}\right)=\partial\left(f_{x, D f(x)}^{\prime \prime}+g_{x, u}^{\prime \prime}\right)$. This subdifferential equation yields $h_{x, z}^{\prime \prime}=f_{x, D f(x)}^{\prime \prime}+g_{x}^{\prime \prime}$. The "only if" part and (4.3) are then proved.

For the "if" part, let

$$
\Delta(\xi)=\left\{\eta: D^{2} f(x) \xi+\eta \in(\partial h)_{x, z}^{\prime}(\xi)\right\}, \quad \xi \in E,
$$

where the proto-derivative $(\partial h)_{x, z}^{\prime}=\mathrm{G}-\lim _{t \downarrow 0} \Phi_{t}$ exists and is a maximal monotone operator by Theorem 3.9. We need to show that $\Delta_{t}$ (the difference quotient of $\partial g$ ) graphically converges to $\Delta$, i.e. $\lim \sup _{t \downarrow 0}\left[\operatorname{gph} \Delta_{t}\right] \subset \operatorname{gph} \Delta \subset$ $\lim \inf _{t \downarrow 0}\left[\operatorname{gph} \Delta_{t}\right]$ or that for every $\xi \in E$,

(i) If $\eta \in \Delta(\xi)$, then for every sequence $t_{n} \downarrow 0$, there exist sequences $\xi_{n} \rightarrow \xi$, $\eta_{n} \in \Delta_{t_{n}}\left(\xi_{n}\right)$ such that $\eta_{n} \rightarrow \eta$.

(ii) If $t_{n} \downarrow 0, \xi_{n} \rightarrow \xi, \eta_{n} \in \Delta_{t_{n}}\left(\xi_{n}\right)$ such that $\eta_{n} \rightarrow \eta$, then $\eta \in \Delta(\xi)$.

To see (i), choose sequences $\xi_{n} \rightarrow \xi, \omega_{n} \in \Phi_{t_{n}}\left(\xi_{n}\right)$ such that $\omega_{n} \rightarrow D^{2} f(x) \xi$ $+\eta$ (this can be done since $\left.\operatorname{gph}(\partial h)_{x, z}^{\prime} \subset \liminf \operatorname{in}_{t \downarrow}\left[\operatorname{gph} \Phi_{t}\right]\right)$. Let $\eta_{n}=\omega_{n}-$ $\Gamma_{t_{n}}\left(\xi_{n}\right)$. Then $\eta_{n} \in \Delta_{t_{n}}\left(\xi_{n}\right)$ by (4.4) and $\eta_{n} \rightarrow \eta$ by (4.5), hence (i) is true.

For (ii), we first see that $\Gamma_{t_{n}}\left(\xi_{n}\right)+\eta_{n} \rightarrow D^{2} f(x) \xi+\eta$ by (4.5) and that $\Gamma_{t_{n}}\left(\xi_{n}\right)+\eta_{n} \in \Phi_{t_{n}}\left(\xi_{n}\right)$ by (4.4). Thus by $\lim \sup _{t \downarrow}\left[\mathrm{gph} \Phi_{t}\right] \subset \operatorname{gph}(\partial h)_{x, z}^{\prime}$, one has $D^{2} f(x) \xi+\eta \in(\partial h)_{x, z}^{\prime}(\xi)$. This shows that $\eta \in \Delta(\xi)$, hence (ii) is true.

We have shown that $\mathrm{G}-\lim \Delta_{t}=\Delta$. Therefore $(\partial g)^{\prime}$ exists and equals to $\Delta$ which is then a monotone operator. The maximality of $\Delta$ follows from that of $(\partial h)_{x, z}^{\prime}$. By Theorem 3.9, $g$ is twice epi-differentiable at $x$ relative to $u$.

Theorem 4.3. Let $E$ be a Hilbert space and $f$ be a closed proper convex function on $E$. Let $\lambda>0$. Then the Moreau-Yosida approximate

$$
f^{\lambda}(x)=\inf _{u \in E}\left\{f(u)+\frac{1}{2 \lambda}\|u-x\|^{2}\right\} \quad(x \in E)
$$


is a $\mathscr{C}^{1}$ function. The infimum above is always attained at a unique point which will be denoted by $J_{f}^{\lambda}(x)$. The mapping $J_{f}^{\lambda}$ is nonexpansive and the function $D f^{\lambda}$ is Lipschitzian with constant $(1 / \lambda)$. One has

$$
\begin{gathered}
u=J_{f}^{\lambda}(x) \Leftrightarrow \frac{1}{\lambda}(x-u) \in \partial f(u), \\
D f^{\lambda}(x)=\frac{1}{\lambda}(x-u) .
\end{gathered}
$$

With $x, u$ and $z=D f^{\lambda}(x)$ as above, the following are equivalent:

(a) $f$ is twice epi-differentiable at $u$ relative to $z$;

(b) $f^{\lambda}$ is twice epi-differentiable at $x$;

(c) $J_{f}^{\lambda}$ is proto-differentiable at $x$ and $\operatorname{dom}\left(J_{f}^{\lambda}\right)_{x, u}^{\prime}=E$;

(d) $J_{f}^{\lambda}$ has one-sided directional derivative at $x$ relative to every $\xi \in E$.

When these hold, one has

$$
\begin{gathered}
\frac{1}{2}\left(f^{\lambda}\right)_{x, z}^{\prime \prime}(\xi)=\left(\frac{1}{2} f_{u, z}^{\prime \prime}\right)^{\lambda}(\xi), \quad \xi \in E, \\
\left(J_{f}^{\lambda}\right)_{x, u}^{\prime}(\xi)=\arg \min _{\eta \in E}\left\{\frac{1}{2} f_{u, z}^{\prime \prime}(\eta)+\frac{1}{2 \lambda}\|\eta-\xi\|^{2}\right\} \\
=\lim _{t \downarrow 0} \frac{J_{f}^{\lambda}(x+t \xi)-J_{f}^{\lambda}(x)}{t}, \quad \xi \in E,
\end{gathered}
$$

where the convergence in the limit above is uniform for $\xi$ in a compact set and $\left(J_{f}^{\lambda}\right)^{\prime}$ is a nonexpansive and positively homogeneous mapping. The condition $\operatorname{dom}\left(J_{f}^{\lambda}\right)_{x, u}^{\prime}=E$ in (c) is superfluous when $E$ is finite-dimensional.

Proof. The properties of $f^{\lambda}, J_{f}^{\lambda}$, and $D f^{\lambda}$ are well-known facts in convex analysis; see [1, Theorems 3.24 and 3.56]. We wish to show (a) $\Leftrightarrow(b)$ and (4.11), (b) $\Leftrightarrow$ (c) and (4.12), and then (c) $\Leftrightarrow$ (d) and (4.13) and the last statement of the theorem.

To show the equivalence of (a) and (b), we note that $f^{\lambda}=f \square \frac{1}{2 \lambda}\|\cdot\|^{2}$ (infimal convolution), so

$$
\left(f^{\lambda}\right)^{*}=f^{*}+\frac{\lambda}{2}\|\cdot\|^{2} .
$$

By Theorem 2.5, $f$ is twice epi-differentiable at $u$ relative to $z$ if and only if $f^{*}$ is twice epi-differentiable at $z$ relative to $u$. From (4.14) and Theorem 4.2, the latter is true if and only if $\left(f^{\lambda}\right)^{*}$ is twice epi-differentiable at $z$ relative to $x=u+\lambda z$ (where $\lambda z$ is the Fréchet derivative of $\frac{\lambda}{2}\|\cdot\|^{2}$ at $z$ ). By Theorem 2.5 again, the above statement is equivalent to the twice epi-differentiability of $f^{\lambda}$ at $x$. Thus (a) and (b) are equivalent. One has

$$
\begin{aligned}
{\left[\frac{1}{2}\left(f^{\lambda}\right)_{x, z}^{\prime \prime}\right]^{*} } & =\frac{1}{2}\left(f^{\lambda}\right)_{z, x}^{* \prime \prime}=\frac{1}{2}\left[\left(f^{*}\right)_{z, u}^{\prime \prime}+\lambda\|\cdot\|^{2}\right] \\
& =\left(\frac{1}{2} f_{u, z}^{\prime \prime}\right)^{*}+\frac{\lambda}{2}\|\cdot\|^{2}=\left(\frac{1}{2} f_{u, z}^{\prime \prime} \square \frac{1}{2 \lambda}\|\cdot\|^{2}\right)^{*},
\end{aligned}
$$

where the first and third equalities come from Theorem 2.5 and the second one comes from (4.14) and Theorem 4.2 (note that the second-order Fréchet derivative of $\frac{\lambda}{2}\|\cdot\|^{2}$ is the function $\left.(\xi, \eta) \mapsto \lambda\langle\xi, \eta\rangle\right)$. Thus

This shows (4.11).

$$
\frac{1}{2}\left(f^{\lambda}\right)_{x, z}^{\prime \prime}=\frac{1}{2} f_{u, z}^{\prime \prime} \square \frac{1}{2 \lambda}\|\cdot\|^{2}=\left(\frac{1}{2} f_{u, z}^{\prime \prime}\right)^{\lambda} .
$$


Next we show the equivalence of (b) and (c). By Theorem 3.9, (b) is equivalent to $D f^{\lambda}$ being proto-differentiable at $x$ with $\left(D f^{\lambda}\right)_{x, z}^{\prime}$ being a maximal monotone operator. Using the Lipschitzian property of $D f^{\lambda}$, we will verify that

$$
\left(D f^{\lambda}\right)_{x, z}^{\prime} \text { is maximal } \Leftrightarrow \operatorname{dom}\left(D f^{\lambda}\right)_{x, z}^{\prime}=E .
$$

In fact, let $\Gamma_{t}$ be the difference quotient of $\left(D f^{\lambda}\right)$ at $x$ relative to $z$. Suppose $\left(D f^{\lambda}\right)_{x, z}^{\prime}$ is maximal. For any $\xi \in E$, the set $\left\{\Gamma_{t}(\xi)\right\}_{t>0}$ is bounded by Lipschitzian property of $D f^{\lambda}$, hence it has a weak limit point $\eta$. By maximality of $\left(D f^{\lambda}\right)_{x, z}^{\prime}$, one has $\eta \in\left(D f^{\lambda}\right)_{x, z}^{\prime}(\xi)$ (cf. [1, Proposition 3.59]). Thus the implication $(\Rightarrow)$ in $(4.15)$ is true. For the converse, it suffices to show the single-valuedness and Lipchitzian property of $\left(D f^{\lambda}\right)_{x, z}^{\prime}$. The verification goes just like that in the proof of Proposition 3.5. Therefore, (4.15) holds.

By (4.15), we have that (b) is equivalent to $\left(D f^{\lambda}\right)$ being proto-differentiable at $x$ with $\operatorname{dom}\left(D f^{\lambda}\right)_{x, z}^{\prime}=E$. The latter is equivalent to $J_{f}^{\lambda}$ being protodifferentiable at $x$ with $\operatorname{dom}\left(J_{f}^{\lambda}\right)^{\prime}=E$. This can be seen as an application of Proposition 3.3 with $\operatorname{gph} J_{f}^{\lambda}=A\left[\operatorname{gph} D f^{\lambda}\right]$, where $A$ is the linear isomorphism $(\xi, \eta) \mapsto(\xi, \xi-\lambda \eta)$ on $E \times E$. Thus (b) and (c) are equivalent. Also, Proposition 3.3 gives

$$
\left(J_{f}^{\lambda}\right)_{x, u}^{\prime}(\xi)=\xi-\lambda\left(D f^{\lambda}\right)_{x, z}^{\prime}(\xi), \quad \xi \in E
$$

From (4.11) one has

$$
\frac{1}{2}\left(f^{\lambda}\right)_{x, z}^{\prime \prime}(\xi)=\inf _{\eta \in E}\left\{\frac{1}{2} f_{u, z}^{\prime \prime}(\eta)+\frac{1}{2 \lambda}\|\eta-\xi\|^{2}\right\}, \quad \xi \in E .
$$

Let $\widetilde{J}(\xi)$ be the right-hand side of (4.12). In view of $(4.8)-(4.10)$, one sees from (4.17) that

$$
\begin{aligned}
\widetilde{J}(\xi) & =\xi-\frac{\lambda}{2} D\left(f^{\lambda}\right)_{x, z}^{\prime \prime}(\xi) \\
& =\xi-\lambda\left(D f^{\lambda}\right)_{x, z}^{\prime}(\xi), \quad \xi \in E(\text { Theorem 3.9). }
\end{aligned}
$$

Thus we obtain (4.12) by comparing (4.16) and (4.18).

It remains to show the equivalence of (c) and (d), (4.13) and the last statement of the theorem. But these are just consequences of Proposition $3.5\left(J_{f}^{\lambda}\right.$ is Lipschitzian). The proof is complete.

Corollary 4.4. Let $C$ be a nonempty closed convex set in a Hilbert space $E$. Let $P=P_{C}$ be the projection mapping on $C$. Let $x \in E$. Then $u=P(x) \Leftrightarrow u \in C$ and $x-u \in N_{C}(u)$. The following are equivalent:

(a) The indicator function $\delta_{C}$ is twice epi-differentiable at $u$ relative to $(x-u)$.

(b) The projection mapping $P$ is proto-differentiable at $x$ with $\operatorname{dom} P_{x, u}^{\prime}=$ $E$.

(c) The projection mapping $P$ has one-sided directional derivative at $x$ relative to every $\xi \in E$. 
When these hold, let $\varphi(\eta)=\frac{1}{2}\left(\delta_{C}\right)_{u, x-u}^{\prime \prime}(\eta), \eta \in E$. Then

$$
P_{x, u}^{\prime}(\xi)=\lim _{t \downarrow 0} \frac{P(x+t \xi)-P(x)}{t} \quad(\xi \in E)
$$

is the solution of the following problem:

$\left(\widetilde{\mathscr{P}_{\xi}}\right) \quad$ Minimize $\varphi(\eta)+\frac{1}{2}\|\eta-\xi\|^{2}$ over $\eta \in E$.

The convergence in (4.19) is uniform for $\xi$ in a compact set and the condition $\operatorname{dom} P_{x, u}^{\prime}=E$ in (b) is superfluous when $E$ is finite-dimensional.

Proof. Just apply the above theorem with $f=\delta_{C}$ and $\lambda=1$.

Example 4.5 (Zarantonello [22, p. 300]). Let $C$ be a closed convex set in a Hilbert space $E$ and $P=P_{C}$ be the projection mapping on $C$. Let $x \in C$ and let $T$ be the tangent cone to $C$ at $x$. Then for any $\xi \in E$,

$$
P(x+t \xi)=x+t P_{T}(\xi)+o(t), \quad t>0 \text {. }
$$

Proof. Apply the above corollary and Example 2.7(b).

Example 4.6 (Haraux [8]). Let $C$ be a polyhedric convex set in a Hilbert space $E$ and $P=P_{C}$ be the projection mapping on $C$. Let $x \in E, u=P(x)$, and

$$
\mathscr{T}=\left\{\eta \in T_{C}(u):\langle x-u, \eta\rangle=0\right\} .
$$

Then for any $\xi \in E$,

$$
P(x+t \xi)=P(x)+t P_{\mathscr{T}}(\xi)+o(t), \quad t>0 .
$$

Proof. Apply the above corollary and Example 2.10.

\section{INTEGRAL FUNCTIONALS}

In this section, we shall see that the twice epi-differentiability of a convex integrand $f: S \times \mathbf{R}^{N} \rightarrow \overline{\mathbf{R}}$ carries over to that of the corresponding integral $I_{f}(x)=\int_{S} f(s, x(x)) d s$ on $x \in \mathscr{L}_{N}^{p}(S) \quad(1<p<\infty)$, the space of $p$-integrable measurable functions $x$ from a measurable space $S$ to $\mathbf{R}^{N}$. This result therefore covers a large class of convex integral functionals, since the twice-epidifferentiability of the integrands are quite easy to obtain; see [13, 14, 17]. The twice epi-differentiability of $I_{f}$ as a function in $\mathscr{L}^{p}$ implies the proto-differentiability of the subdifferential mapping $\partial I_{f}$ (Theorem 3.9). The result could then be applied to analyze solutions of optimization problems as worked out in [7].

Throughout the section, the set $S$ is a nonempty measurable space with $\sigma$ algebra $\mathscr{A}$ and a $\sigma$-finite measure $d s$; typically, $S$ is a closed interval $\left[s_{0}, s_{1}\right]$ in $\mathbf{R}$ with Lebesgue measure. The space $\mathscr{L}_{N}^{p}(S)$ is usually written as $\mathscr{L}_{N}^{p}$, (the set $S$ is omitted) or as $\mathscr{L}^{p}$, for simplicity.

Definition 5.1. A closed-valued multifunction $\Gamma: S \rightrightarrows \mathbf{R}^{N}$ is said to be measurable if the set $\Gamma^{-1}(U)=\{s \in S: \Gamma(s) \cap U \neq \varnothing\}$ is measurable for every open set $U \subset \mathbf{R}^{N}$. An integrand $f: S \times \mathbf{R}^{N} \rightarrow \overline{\mathbf{R}}$ is said to be $\mathscr{A}$-normal (or simply normal if there is no confusion) if its epigraph epi $f(s, \cdot)$ as a function of $s$ is a closed-valued measurable multifunction: $S \rightrightarrows \mathbf{R}^{N}$. The closed-valuedness of 
$s \mapsto$ epi $f(s, \cdot)$ is equivalent to $f(s, \cdot)$ being lower semicontinuous for all $s$. Furthermore, $f$ is said to be a convex integrand if $f(s, \cdot)$ is a convex function for a.e. $s \in S$. A normal integrand $f$ is called proper if $f(s, \cdot)>-\infty$ and $f(s, \cdot) \not \equiv \infty$ for a.e. $s \in S$. An integrand $f$ is called a Carathéodory integrand if $f(s, x)$ is finite, measurable in $s$, and continuous in $x$ for a.e. $s \in S$. If the latter two properties hold for $f: S \times \mathbf{R}^{N} \rightarrow \mathbf{R}^{m}$, we call $f$ a Carathéodory mapping.

For a thorough treatment of normal integrands, we refer the reader to [15] from which we extract a few results here:

(M1) A closed-valued multifunction $\Gamma$ is measurable if and only if the distance $d(x, \Gamma(s))$ is a measurable function of $s \in S$ for every $x \in \mathbf{R}^{N}$.

(M2) If $f$ is a normal integrand and $x$ is a measurable function, then $s \mapsto$ $f(s, x(s))$ is a measurable function.

(M3) A positive linear combination of normal proper integrands is a normal proper integrand.

(M4) If $\varphi(s, \eta)=f(s, h(s, \eta))$ where $f$ is a normal integrand and $h$ is a Carathéodory mapping, then $\varphi$ is a normal integrand.

An example of the kind of mapping $h$ for which (M4) is intended is $h(s, \eta)=$ $(\eta, \xi(s))$ where $\xi: S \rightarrow \mathbf{R}^{m}$ is a measurable function.

(M5) If $f$ is a normal convex integrand, then so is $f^{*}$. (The conjugacy is taken with respect to the second argument, $x$.)

For a measurable function $\varphi$ on $S$, let $\varphi^{+}, \varphi^{-}$be the positive and negative parts of $\varphi$ respectively, i.e. $\varphi^{+}=\max \{0, \varphi\}$ and $\varphi^{-}=\max \{0,-\varphi\}$. Then $\int_{S} \varphi^{+}$and $\int_{S} \varphi^{-}$are well defined as an element in $[0, \infty]$. The fact $\int_{S} \varphi^{+}<\infty$ is equivalent to $\varphi$ being majorized by a summable function. Likewise, $\int_{S} \varphi^{-}<$ $\infty$ is equivalent to $\varphi$ being minorized by a summable function. In either case, the integral

$$
\int_{S} \varphi=\int_{S} \varphi^{+}-\int_{S} \varphi^{-}
$$

is well defined as an element in $\overline{\mathbf{R}}$. If both cases fail, we assign $\infty$ value to $\int_{S} \varphi$, or equivalently, $\int_{S} \varphi$ is given in (5.1) under the convention $\infty-\infty=\infty$. Under this convention, if $f$ is a normal convex integrand, then the integral functional $I_{f}(x)=\int_{S} f(s, x(s)) d s\left(x \in \mathscr{L}^{p}\right)$ is a convex function of $x$.

We shall only be interested in the case that $I_{f}$ is proper, i.e. $I_{f}$ never takes on $-\infty$ and $I_{f}(x)<\infty$ for some $x \in \mathscr{L}^{p}$. In such case, we can see that the ambiguity $\infty-\infty$ never occurs in $\int_{S} f(s, x(s)) d s$ when using the expression of (5.1), in other words, $\int_{S} f(s, x(s)) d s$ is well defined in the usual sense for every $x \in \mathscr{L}^{p}$.

Proof. In fact, suppose there were an $x_{1} \in \mathscr{L}^{p}$ such that

$$
\int_{S} f\left(s, x_{1}(s)\right)^{+} d s=\int_{S} f\left(s, x_{1}(s)\right)^{-} d s=\infty .
$$

By properness of $I_{f}$, we can pick an $x_{0} \in \mathscr{L}^{p}$ such that $I_{f}\left(x_{0}\right)$ is finite. Let

$$
\begin{aligned}
A^{+}=\left\{s: f\left(s, x_{1}(s)\right) \geq 0\right\}, & A^{-}=\left\{s: f\left(s, x_{1}(s)\right)<0\right\}, \\
\bar{x}(s) & = \begin{cases}x_{0}(s) & \text { if } s \in A^{+}, \\
x_{1}(s) & \text { if } s \in A^{-} .\end{cases}
\end{aligned}
$$


Then $\bar{x} \in \mathscr{L}^{p}$ and

$$
\int_{S} f(s, \bar{x}(s))^{+} d s<\infty, \quad \int_{S} f(s, \bar{x}(s))^{-} d s=\infty .
$$

This would imply that $I_{f}(\bar{x})=-\infty$, a contradiction.

We now turn to the twice epi-differentiability of the integral functionals. First, we note that the normality of integrands is preserved under the epiconvergence:

(M6) Let $\varphi_{t}: S \times \mathbf{R}^{N} \rightarrow \overline{\mathbf{R}}$ be a normal integrand for each $t>0$. Assume that there exists a $\varphi: S \times \mathbf{R}^{N} \rightarrow \overline{\mathbf{R}}$ such that $\varphi_{t}(s, \cdot)$ epi-converges to $\varphi(s, \cdot)$ (as $t \downarrow 0$ ) for a.e. $s \in S$. Then $\varphi$ is a normal integrand.

Proof. Let $\Gamma_{t}(s)=\operatorname{epi} \varphi_{t}(s, \cdot), \Gamma(s)=\operatorname{epi} \varphi(s, \cdot)$. Then by hypothesis, $\Gamma_{t}(s)$ epi-converges to $\Gamma(s)$ as $t \downarrow 0$, for a.e. $s \in S$. This implies that $\Gamma(s)$ is closed for a.e. $s$. We need to show that $\Gamma$ is a measurable multifunction.

Each $\Gamma_{t}$ is a measurable multifunction, hence by property (M1) the function $s \mapsto d\left(x, \Gamma_{t}(s)\right)$ is measurable for every $x \in \mathbf{R}^{N}$. Since $d(x, \Gamma(s))=$ $\lim _{t \downarrow 0} d\left(x, \Gamma_{t}(s)\right)$ (note that this holds in the finite-dimensional case without any convexity assumption), we have that $s \mapsto d(x, \Gamma(s))$ is a measurable function for every $x \in \mathbf{R}^{N}$. By property (M1) again, we see that $\Gamma$ is a measurable multifunction.

Lemma 5.2. Let $f: S \times \mathbf{R}^{N} \rightarrow \overline{\mathbf{R}}$ be a normal proper integrand. Let $x \in \mathscr{L}^{p}$ and assume that $f(s, x(s))$ is finite for a.e. $s \in S$. Then for each $t>0$ and each $z \in \mathscr{L}^{p}$ the second-order difference quotient function

$$
\begin{aligned}
\varphi_{t}(s, \xi) & :=\left(\Delta_{t}^{(2)} f\right)_{x(s), z(s)}(s, \xi) \\
& =\frac{2}{t^{2}}[f(s, x(s)+t \xi)-f(s, x(s))-t z(s) \cdot \xi]
\end{aligned}
$$

is a normal proper integrand.

Proof. Write $\varphi_{t}=\frac{2}{t^{2}}\left[f_{1}+f_{2}+f_{3}\right]$, where

$$
f_{1}(s, \xi)=f(s, x(s)+t \xi), \quad f_{2}(s, \xi)=-f(s, x(s)), \quad f_{3}(s, \xi)=-t z(s) \cdot \xi .
$$

The functions $f_{2}$ and $f_{3}$ are Carathéodory integrands, $f_{3}$ is normal proper by property (M4), hence $\varphi_{t}$ is normal proper by property (M3).

Definition 5.3. A sequence $\left(\varphi_{n}\right)$ of normal integrands is said to be $p$-bounded $(1<p<\infty)$ in the sense of Joly and Thenin [10] if there exist a sequence $\left(x_{n}\right)$ in $\mathscr{L}^{p}$, an element $\alpha$ in $\mathscr{L}^{p}$, and an element $\gamma$ in $\mathscr{L}^{1}$ such that

(i) $\left|x_{n}(s)\right| \leq \alpha(s)$ for a.e. $s$,

(ii) $\varphi_{n}\left(s, x_{n}(s)\right) \leq \gamma(s)$ for a.e. $s$.

Theorem 5.4 [10, Theorem 1]. Let $\left(\varphi_{n}\right)$ be a sequence of normal convex integrands. Suppose that $\left(\varphi_{n}\right)$ is p-bounded $(1<p<\infty),\left(\varphi_{n}^{*}\right)$ is q-bounded $\left(\frac{1}{p}+\frac{1}{q}=1\right)$, and $\left(\varphi_{n}\right)$ epi-converges to $\varphi$. Then $I_{\varphi_{n}}, I_{\varphi_{n}^{*}}, I_{\varphi}, I_{\varphi^{*}}$ are proper convex functions and $I_{\varphi_{n}} \stackrel{M}{\rightarrow} I_{\varphi}, I_{\varphi_{n}^{*}} \stackrel{M}{\rightarrow} I_{\varphi^{*}}$.

Theorem 5.5. Let $1<p<\infty$ and let $f: S \times \mathbf{R}^{N} \rightarrow \overline{\mathbf{R}}$ be a normal proper convex integrand and suppose that $I_{f}$ is proper. Then

(a) $z \in \partial I_{f}(x) \Leftrightarrow z \in \mathscr{L}^{p}$ and $z(s) \in \partial f(s, x(s))$ for a.e. $s \in S$. 
(b) With $x, z$ as in (a), assume that $f(s, \cdot)$ is twice epi-differentiable at $x(s)$ relative to $z(s)$ for a.e. $s \in S$. Then $I_{f}$ is twice epi-differentiable at $x$ relative to $z$ and

$$
\left(I_{f}\right)_{x, z}^{\prime \prime}(\xi)=\int_{S} f_{x(s), z(s)}^{\prime \prime}(s, \xi(s)) d s, \quad \xi \in \mathscr{L}^{p}
$$

Proof. Assertion (a) is a special case of [15, Corollary 3E]. To prove (b), let $\varphi_{t}(s, \cdot)=\left(\Delta_{t}^{(2)} f\right)_{x(s), z(s)}(s, \cdot)$ be the second order difference quotient function of $f$ and $\varphi(s, \cdot)=f_{x(s), z(s)}^{\prime \prime}(s, \cdot)$ be the second-order epi-derivative. By Lemma $5.2, \varphi_{t}$ is a normal integrand, and by hypothesis, $\varphi_{t}(s, \cdot)$ epi-converges to $\varphi(s, \cdot)$. Since

$$
\varphi_{t}(s, \cdot) \geq 0, \quad \varphi_{t}(s, 0)=0, \quad \text { for a.e. } s \in S,
$$

we have $\varphi_{t}^{*}(s, 0)=0$ for a.e. $s$. Hence $\left(\varphi_{t}\right)$ is $p$-bounded and $\left(\varphi_{t}^{*}\right)$ is $q$-bounded. Thus by Theorem 5.4, $I_{\varphi_{t}}$ Mosco epi-converges to $I_{\varphi}$.

Observe that $I_{\varphi_{t}}$ is the second-order difference quotient function $\left(\Delta_{t}^{(2)} I_{f}\right)_{x, z}$ of $I_{f}$, and $I_{\varphi}$ is the right-hand side of (5.2), part (b) then follows.

\section{REFERENCES}

1. H. Attouch, Variational convergence for functions and operators, Pitman, London, 1984

2. H. Attouch, D. Azé and R. J.-B. Wets, Convergence of convex-concave saddle functions: continuity properties of the Legendre-Fenchel transform. Applications to convex programming and mechanics, Ann. Inst. Henri Poincaré 5 (1988), 537-572.

3. A. BenTal and J. Zowe, Necessary and sufficient conditions for a class of nonsmooth minimization problems, Math. Programming 24 (1982), 70-91.

4. R. W. Chaney, Second-order sufficient conditions for nondifferentiable programming problems, SIAM J. Control Optim. 20 (1982), 20-33.

5. F. Clarke, Optimization and nonsmooth analysis, Wiley, New York, 1983.

6. Chi N. Do, Second-order nonsmooth analysis and sensitivity in optimization problems involving convex integral functionals, PhD. Thesis, Univ. of Washington, 1989.

7. __ Sensitivity analysis in optimal control problems with convex costs, preprint.

8. A. Haraux, How to differentiate the projection on a convex set in Hilbert space: some applications to variational inequalities, J. Math. Soc. Japan 29 (1977), 615-631.

9. J.-B. Hiriart-Urruty, Calculus rules on the approximate second-order directional derivative of a convex function, SIAM J. Control Optim. 22 (1984), 381-404.

10. Jean-Luc Joly and François de Thelin, Convergence of convex integrals in $\mathscr{L}^{p}$ spaces, J. Math. Anal. Appl. 54 (1976), 230-244.

11. J. L. Ndoutoume, Calcul différentiel généralisé du second ordre, preprint.

12. R. Phelps, Convex functions, monotone operators and differentiability, Springer-Verlag, 1989.

13. R. T. Rockafellar, First and second-order epi-differentiability in nonlinear programming, Trans. Amer. Math. Soc. 307 (1988), 75-108.

14. _ Generalized second derivatives of convex functions and saddle functions, Trans. Amer. Math. Soc. 28 (1990), 810-822.

15. Integral functionals, normal integrands and measurable selections, Nonlinear Operators and the Calculus of Variations, Springer-Verlag, 1976, pp. 157-207.

16. __ Proto-differentiability of set-valued mappings and its applications in optimization, Analyse Non Linéaire (H. Attouch et al., eds.), Gauthier-Villars, Paris, 1989, pp. 449-482.

17. derivatives, Math. Oper. Res. 14 (1989), 462-484. 
18. G. Salinetti and R. J.-B. Wets, Convergence of sequences of closed sets, Topology Proc. 4 (1979), 149-157.

19. _ On the convergence of sequences of convex sets in finite dimensions, SIAM Rev. 21 (1979), 18-33.

20. A. Seeger, Second-order directional derivatives in parametric optimization problems, preprint.

21. J. Sokolowski, Differential stability of solutions to constrained optimization problems, Appl. math. Optim., Springer-Verlag, 1985, pp. 97-115.

22. E. H. Zarantonello, Projections on convex sets in Hilbert space and spectral theory, Contributions to Nonlinear Functional Analysis, 1971, pp. 237-424.

Department of Mathematics, University of Rhode Island, Kingston, Rhode Island 02881

Current address: 5110 S. 300 Place, Auburn, Washington 98001 